\title{
Calibration Method for an Inductive Localization System of Wireless Sensors in Photoreactors
}

\author{
David Demetz ${ }^{1}$, Alexander Sutor ${ }^{2}$ \\ ${ }^{1}$ Institute of Measurement and Sensor Technology, UMIT - Private University, Hall in Tirol, Austria \\ david.demetz@umit.at \\ 2 Institute of Measurement and Sensor Technology, UMIT - Private University, Hall in Tirol, Austria \\ alexander.sutor@umit.at
}

\begin{abstract}
Summary:
We present a calibration method for an inductive localization system used to trace wireless sensors for the future use in photoreactors. Photoreactors are used to cultivate photosynthetic active microorganisms and cells or to perform photocatalytic reactions. Due to the limited penetration depth of light inside those reactors, a novel internal illumination system have been presented in past. The illumination system consists of so called Wireless light emitters (WLE) which are small glowing spheres floating around in the reactor. The WLE are powered through an inductive link where the driving coils are placed at the outer diameter of the reactor. They produce an intermediate frequency $(178 \mathrm{kHz})$ electromagnetic field with a magnetic flux density of approx. B = $1 \mathrm{mT}$ [1] - [3]. The next step in this project is the inclusion of sensors for measuring various parameters inside the reactor. Additionally, the information about the sensor position leads to a spatial resolution of the measured quantity. Therefore, wireless sensors are needed as well as a suited localization method.
\end{abstract}

Keywords: inductive communication, inductive localization, calibration method, wireless sensors

\section{Introduction}

To control the processes in those photoreactors various parameters have to be measured e.g. temperature, $\mathrm{pH}$-value, UV-illumination or other chemical concentrations. To avoid the drawback of measuring the named parameters always at the same position inside the reactor, wireless traceable sensors are being developed. Because of the promising propagation properties of magnetic fields in water and in salty water, we chose the inductive layer for the communication and the localization task. The authors of [4] also make use of this advantage for the inductive communication through human tissue for assistive listening devices. For our photoreactor use, we set the modulation frequency of the data transmission link $(297 \mathrm{kHz})$ at a factor 1.66 above the frequency of the WLE power link in order to prevent interferences by harmonics. The drawback of using higher frequencies would be their high damping factor in electrically conducting media.

\section{Inductive communication and localization}

As a modulation technique for the data transmission, we use the on-off-keying (OOK); this is implemented like in [4] with an on-off switched hartley-oscillator as transmitter. In this first step, we do not use real sensors. We use a square wave generator based on the integrated circuit
LMC555 to simulate the digital sensor data stream. We already presented the electrical circuit of the mentioned transmitter before [5]. To solve the localization task, we make use of the well-defined spatial propagation properties of the magnetic dipole field, which is used to describe the field of our transmitting coil. At a frequency of $297 \mathrm{kHz}$, the wavelength is big compared to the distances between the transmitter and the receivers in our setup. Therefore, only the quasistatic field component must be considered [6]. In the publication [7] we already presented the analytical coupling equation system used to calculate the directional vectors, which point to the transmitter position from every measuring point of its magnetic field. The transmitter position is calculated by finding the point where two or more directional vectors comes closest to each other (the intersection in an ideal case).

\section{Receiver design and setup}

The main requirement to our receivers is the ability to measure all three spatial components of the transmitter magnetic field. This requirement is satisfied by using three coils placed orthogonally to each other. This design minimalizes the mutual inductions between them. Since each coil is directly connected to the input of an operational amplifier, no current flows through them. This fact additionally ensures that the receiver coils 
do not influence each other. The received signals are filtered with operational amplifier based amplifiers and active filters and are then digitalized by the I/O device National Instruments USB6366. The software Matlab by MathWorks is used to control the I/O device and for solving the coupling equation in order to calculate the directional vectors and consequently the transmitter position. We build a setup for performing inductive localization measurements in which two receivers are each placed in a corner of our cubic room of interest (ROI). This is needed because by solving the coupling equation due to the symmetry properties of the magnetic dipole field, we get multiple possible solutions for the directional vector. By placing the receiver in a corner of the ROI, the multiple solutions are automatically diminished to one right solution. This because the other solutions would not point into the ROI. The size of our ROI is $50 \mathrm{~cm}$ in width and height and $30 \mathrm{~cm}$ in depth.

\section{Receiver calibration}

The receivers are calibrated using a Lidar depth Camera. Therefore, we chose the Intel Realsense Lidar Camera L515. The camera is mounted above the ROI pointing downwards in order to capture the whole setup. According to its datasheet, the depth accuracy of the camera is lower than $5 \mathrm{~mm}$ at VGA (640 per 480 pixel) resolution and a maximal distance of $1 \mathrm{~m}$. In our setup, the maximal distances are always under $1 \mathrm{~m}$. Additionally, the depth camera is used with the resolution set to XGA (1024 per 768 pixel) which further increases its depth accuracy. For the calibration, both systems are used to locate the transmitting coil; the depth camera and the inductive system. The position measured with the camera is considered to be the correct one. In order to calibrate the receivers, the transmitter position measured with the depth camera is used to calculate the amplitudes in each receiver coil. A comparison between the normalized measured receiver amplitudes and the normalized calculated amplitudes enables an adjustment of the gain factor of each coil signal amplifier. Since the used transmitter coil does not have ideal properties, the calibration of our system needs a second step. Due to inaccuracies in winding the coil by hand, the magnetic dipole equation does not accurately model our real coil. Even if the distance between the transmitter and the receivers is big compared to the coil dimensions, the system needs some further adjustments. Therefore, a defined grid of points are selected in the ROI. The transmitter coil is placed on each of those points and its position is measured with the depth camera as well as with the inductive localization system. We calculate a displacement vector between the correct position and the inductively measured position for each grid point.
Those vectors are saved in a so called displacement map. We use the map in a second step to tune each further inductive localization measurements by adjusting the measured position by means of the displacement vectors from the map.

\section{Discussion and outlook}

The feasibility of the described calibration method has been tested with some first test measurements. We were able to observe a clear improvement in the localization accuracy. The next step is the registration of a good resolved calibration map in order to best increase the overall accuracy. The recorded calibration map can also be used to analyze the influence of e.g. nearby standing electrically conducting materials that would affect the magnetic field and so the accuracy of our localization system. This can be done by comparing it to a second calibration map with the same grid points registered with the interfering objects near the ROI.

\section{References}

[1] M. Heining, A. Sutor, S. Stute, C. Lindenberger und R. Buchholz, "Internal illumination of photobioreactors via wireless light emitters:", J ournal of Applied Phycology, vol. 27, pp. 59-66, 2015, doi:10.1007/s10811-014-0290-x.

[2] A. Sutor, M. Heining und R. Buchholz, „A ClassE Amplifier for a Loosely Coupled Inductive Power," Energies, Bd. 12, Nr. 6, 2019, doi: 10.3390/en12061165.

[3] B. O. Burek, A. Sutor, B. W. Bahnemann und J . Z. Bloh, „Completely integrated wirelesslypowered photocatalyst-coated spheres as a novel means to perform heterogeneous photocatalytic reactions," Catal. Sci. Technol., Bd. 7, Nr. 21, p. 4977-4983, 2017, doi: 10.1039/c7cy01537b.

[4] J . Edelmann, R. Stojakovic, C. Bauer und T. Ussmueller, „An Inductive Through-The-Head OOK Communication Platform for Assistive Listening Devices," 2018 IEEE Topical Conference on Wireless Sensors and Sensor Networks (WiSNet), p. 30-33, 2018, doi: 10.1109/WISNET.2018.8311556.

[5] D. Demetz, O. Zott und A. Sutor, „Wireless and Traceable Sensors for Internally Illuminated Photoreactors," Proceedings of the IEEE International Conference on Industrial Technology (ICIT), pp. 582-286, 2020, isbn: 978 1-7281-5754-2.

[6] F. H. Raab, E. B. Blood, T. O. Steiner und H. R. Jones, „Magnetic position and orientation tracking system," IEEE Transactions on Aerospace and Electronic Systems, p. 709-718, 1979, doi: 10.1109/TAES.1979.308860.

[7] D. Demetz und A. Sutor, „Inductive Communication and Localization of Wireless Sensors in Photoreactors," SMSI 2020 Measurement Science, pp. 325 - 326, 2020, doi: 10.5162/SMSI2020/P3.5. 